AperTO - Archivio Istituzionale Open Access dell'Università di Torino

\title{
Self-assembly drug conjugates for anticancer treatment
}

\section{This is the author's manuscript}

Original Citation:

Availability:

This version is available http://hdl.handle.net/2318/1616905

since 2017-05-18T13:52:40Z

Published version:

DOI:10.1016/j.drudis.2016.06.018

Terms of use:

Open Access

Anyone can freely access the full text of works made available as "Open Access". Works made available under a Creative Commons license can be used according to the terms and conditions of said license. Use of all other works requires consent of the right holder (author or publisher) if not exempted from copyright protection by the applicable law. 


\section{Accepted Manuscript}

Title: Self-assembly drug conjugates for anticancer treatment

Author: Gaia Fumagalli Cristina Marucci Michael S.

Christodoulou Barbara Stella Franco Dosio Daniele Passarella

PII: $\quad$ S1359-6446(16)30236-7

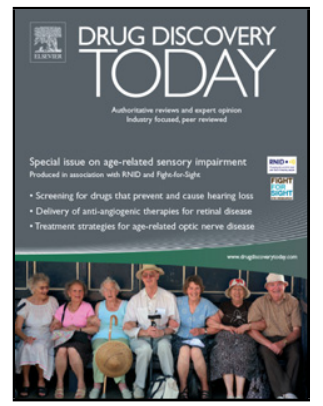

DOI: $\quad$ http://dx.doi.org/doi:10.1016/j.drudis.2016.06.018

Reference: $\quad$ DRUDIS 1844

To appear in:

Received date: $\quad 13-1-2016$

Revised date: $\quad 10-5-2016$

Accepted date: $\quad$ 15-6-2016

Please cite this article as: Fumagalli, G., Marucci, C., Christodoulou, M.S., Stella, B., Dosio, F., Passarella, D.,Self-assembly drug conjugates for anticancer treatment, Drug Discovery Today (2016), http://dx.doi.org/10.1016/j.drudis.2016.06.018

This is a PDF file of an unedited manuscript that has been accepted for publication. As a service to our customers we are providing this early version of the manuscript. The manuscript will undergo copyediting, typesetting, and review of the resulting proof before it is published in its final form. Please note that during the production process errors may be discovered which could affect the content, and all legal disclaimers that apply to the journal pertain. 
Self-assembling inducer Linker Drug $\longrightarrow$

Self-assembly

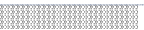

Page 1 of 25 


\section{Highlights:}

- Many anticancer compounds are unable to demonstrate pharmacological activity because they do not reach therapeutic concentration at the site of action

- Nanotechnology is a useful tool to improve the selective delivery of anticancer compounds to their site of action

- An interesting application of anticancer nanotechnology is represented by nanostructures spontaneously obtained by self-assembly of drug conjugates linked to a proper chemical entity

- The generation of hetero-NPs could be exploited for personalized combined treatment of different types of diseases 


\title{
Self-assembly drug conjugates for anticancer treatment
}

\author{
Gaia Fumagalli ${ }^{1}$, Cristina Marucci ${ }^{1}$, Michael S. Christodoulou ${ }^{1}$, Barbara Stella ${ }^{2}$, Franco \\ Dosio $^{2}$ and Daniele Passarella ${ }^{1, *}$
}

${ }^{1}$ Dipartimento di Chimica, Università degli Studi di Milano, Via Golgi 19, 20133 Milano, Italy

${ }^{2}$ Dipartimento di Scienza e Tecnologia del Farmaco, Università degli Studi di Torino, Via Giuria 9, 10125 Torino, Italy

"Corresponding author: Passarella, D. (daniele.passarella@unimi.it).

Keywords: Self-assembly; drug conjugate; nanoparticle.

Teaser. In this review we summarize the recent advances in nanoparticles obtained by self-assembly drug conjugates, a useful tool to improve drug delivery of anticancer compounds. 
Self-assembly drug conjugate preparation is a promising approach to improve activity and penetration through physiological barriers of potent small molecules, as well as to reduce any side effects. Drug conjugates can self-assemble in water to form nanoparticles (NPs) that offer several advantages because: (i) they are easy to obtain; (ii) they can reach high local drug concentration in tumor tissues; and (iii) they can reduce the side effects of drugs. All these factors improve drug pharmacokinetic properties. Here, we have reviewed the scope of nanotechnology-based self-assembly drug delivery approaches focusing on prodrugs able to form NPs by self-assembly; we have also summarized the current perspective and challenges facing the successful treatment of cancer. 


\section{Introduction}

Many anticancer candidates are not able to demonstrate pharmacological activity despite their potent cytotoxicity because a minimum concentration is not reached at the site of action. In fact, most anticancer drugs have their targets within cells and tissues and the low partition coefficient of drugs can prevent the cell barrier permeation and consequently their cytotoxic effect. In recent years, nanotechnology has opened new perspectives for biological and biomedical applications to improve the selective delivery of anticancer compounds to their site of action. The use of nanotechnology in cancer treatment has some advantages [1]; in fact tumor blood vessels are highly disorganized because they are characterized by an inefficient lymphatic network and endothelial cells are less tight compared with normal tissues. Nanoparticles (NPs) can be preferentially delivered to the tumor site because of the enhanced permeation and retention (EPR) effect $[2,3]$. In addition to that, nanotechnologies can improve drug properties in several ways: by controlling release and distribution, by enhancing drug absorption and by protecting the drug from degradation. Micelles, liposomes, nanospheres, nanocapsules, nanorods, nanofibers, etc. are commonly used to load the drugs in the inner core or into the bilayer. A small but interesting niche inside anticancer nanotechnology is represented by nanosystems spontaneously obtained by self-assembly of conjugates composed of drugs linked to a proper chemical entity. The self-assembly of drug conjugates into NPs is a process that involves the formation of an ordered structure by spontaneous organization of building blocks as a consequence of specific local interactions. Assembly can be obtained with different methods and conditions but the key role of the amphilicity of the components is often fundamental.

In recent years, the use of drug conjugates to obtain NPs has gained considerable attention [4-6]. These conjugates are usually obtained by a covalent coupling of the drug to biocompatible lipid moieties and the resulting molecules are able to form NPs by selfassembly. NPs are usually characterized by different techniques to be easily compared to each other and to predict their biological efficiency [7]. Crucial parameters for NP characterization are the mean diameter, which influences their biodistribution and retention, and the surface charge, which is responsible for the interactions with the environment and 
physical and chemical stability. Electron microscopy, dynamic light scattering (DLS), NP tracking analysis (NTA) and disk centrifugation are techniques that rely on different physical principles and sample preparation useful for particle size determination. The surface charge of NPs (zeta potential) is generally measured by laser doppler electrophoresis, which evaluates electrophoretic mobility of suspended NPs in the medium.

The aim of this paper is to review the latest literature (2009-2015) about prodrugs that can form NPs by self-assembly with a specific focus on the functionalization of anticancer compounds. We discuss the characteristics of self-assembly inducers and drug modification and NP biological evaluation. Figures 1 and 2 [s1]represent the structures of the mentioned self-assembly inducers and of the drugs, respectively. In Table 1 we summarize the mentioned drugs, the linker, the used self-assembly inducer and the corresponding characteristics of the obtained nanostructures together with the cancer cell lines used for the biological evaluation. Table 1 also shows the cases in which the in vivo tests were reported.

\section{Self-assembly inducers}

Self-assembly is a process that is mediated by noncovalent interactions between molecules via ionic bonds, $\mathrm{H}$-bonding, hydrophobic interactions and van der Waals interactions. Different organic molecules, such as biocompatible polymeric chains or endogenous molecules (terpenes or polysaccharides), have been used as self-assembly inducers by covalent linkage to drugs. The drug and the self-assembly inducer can be directly attached or can be connected by a linker, which can be stable in serum but able to release the drug intracellularly. In the literature there are different examples detailing the use of biocompatible polymeric chains as self-assembly inducers for the formation of NPs.

\section{Hydrophilic hyperbranched poly(ether-ester)}

The use of biocompatible hydrophilic hyperbranched poly(ether-ester) (HPEE, 1, Figure 1) for the construction of an amphiphilic copolymer with the hydrophobic drug paclitaxel (7, Figure 2) has been reported recently. This conjugate is able to self-assemble in water to form NPs with a diameter of $50-120 \mathrm{~nm}$. The copolymer paclitaxel-HPPE NPs are biodegradable 
and they can be easily eliminated through excretion pathways in vivo because of the presence of many ester groups in the HPPE structure [8].

\section{Polyethylene glycol}

In another study, a multiarm-polyethylene glycol (PEG, 2) was employed for the delivery of anticancer drugs by the formation of self-assembled NPs. A folate-eight-arm PEG-betulinicacid was synthesized[s2] by connection of the poor water-soluble drug betulinic acid (8) (hydrophobic part), PEG (hydrophilic segment) and folate (target molecule). Also in this case the biocompatibility of the NPs is caused by the ester bonds in the conjugate that guarantee the drug release inside the cell. Multiarm-PEGs have some advantages compared with linear PEG such as the increase in molecular weight (low molecular weight PEG had been reported to be toxic for the kidneys) and the presence of many functional groups useful to improve the drug-loading capacity [9].

The use of a linker between the self-assembly inducer and the drug is useful to guarantee the release of the active molecule. In 2010, the covalent conjugation of a Pt(IV) prodrug (9) to a self-assembly inducer through an acid-responsive linker was exploited for the formation of NPs to improve the therapeutic efficacy of compound 9. In this case, a PEG-b-poly(Llactide) copolymer was used to induce self-assembly because of the advantages of polymeric NPs. These NPs can prolong the half-life of drugs in the systemic circulation, limit drugs side effects and release drugs in a controlled manner. The acid-responsive bond favors the control of the drug release in an environmentally sensitive manner. This approach would be useful to minimize the release of the drug in the blood $(\mathrm{pH} \mathrm{7.4)}$ and to facilitate the release inside the cells ( $\mathrm{pH} 5-6)$. The prodrug conjugate, characterized by the presence of an acid-responsive hydrazone bond, was synthesized and it was demonstrated to form NPs by self-assembly via a precipitation method [10]. These NPs showed well-controlled drugloading yield, excellent acid-responsive drug-release properties and potent cytotoxic activity against ovarian cancer [11].

Recently, two different self-assembly inducers covalently bonded have been used to form NPs: a glycol chitosan chain (3) and a PEG block. In addition, the synthesized conjugate 
consists of a functional 3-diethylaminopropyl isothiocyanate (DEAP) block and a photosensitizing drug, chlorine e6 (10). This conjugate self-organized in aqueous solution and some studies revealed that the glycol chitosan and PEG blocks are on the hydrophilic outer shell, and chlorine e6 is within the hydrophobic inner core. The advantages of using these self-assembly inducers are that a polysaccharide-drug conjugate is expected to provide an effective cancer therapy without affecting the safety of normal tissues and a PEG block could improve the stability of the drug conjugate in serum and the penetration into an in vivo tumor vasculature. In addition, this conjugate is characterized by the presence of a $\mathrm{pH}$ sensitive functional group that allows the release of the photosensitive drug in acidic conditions. In fact, it was previously shown that the $\mathrm{pK}_{\mathrm{b}}$ value of a glycol chitosan conjugate with DEAP is near 6.8. In this study it was demonstrated that, upon encountering the tumor environment, the diameter and the zeta potential of NPs change because of a variation in the $\mathrm{pH}$ value from 7.4 to 6.8 . Also, the shape of NPs is affected by the $\mathrm{pH}$ variation - they are spherical under neutral conditions and become disentangled at pH 6.8 [12].

PEG was also used to induce self-assembly in a conjugate with beclin-1 (11), an autophagyinducing peptide. In particular, the amphiphilic poly( $\beta$-amino ester) copolymer used was composed of a hydrophobic monomer 1,6-hexanediol diacrylate, a pH-sensitive monomer 3(dibutylamino)-1-propylamine and a hydrophilic amino-terminated PEG. This copolymer was synthesized by Michael addition and characterized by ${ }^{1} \mathrm{H}-\mathrm{NMR}$. The amphiphilic PEG-beclin1 polymers simultaneously self-assembled during dialysis from dimethyl sulfoxide into water into micelle-like NPs with a mean diameter of $32 \mathrm{~nm}$. The efficacy of the $\mathrm{pH}$-sensitive linker was confirmed by diameter measurements in different $\mathrm{pH}$ conditions - the dimensions of NPs increase considerably, implying that NPs disassociate and reorganize into larger entities under weakly acidic conditions [13].

Du et al. described the use of PEG as a self-assembly inducer in the synthesis of a dual pHsensitive polymer-doxorubicin (12) conjugate. The polyphosphoester was chosen as selfassembly inducer because of its biodegradability and its previous uses in biomedical fields. The parental diblock copolymer monomethoxyl PEG-b-poly-(allylethylene phosphate) was easily prepared by ring-opening polymerization. The obtained conjugate self-assembled to 
give nanostructures in aqueous solution. To prove the efficacy of the $\mathrm{pH}$-sensitive linker, the NPs were incubated at different $\mathrm{pH}$ values and the release of the free drug was monitored. Varying the $\mathrm{pH}$ from 7.4 to 5 , the release of doxorubicin increased markedly indicating the sensitivity of NPs to endo and/or lysosomal $\mathrm{pH}$. This suggests also that NPs can reduce premature drug release during circulation but specifically enhance intracellular drug release, which will be an important feature in cancer treatment [14].

In 2010, the use of an oligomer chain of ethylene glycol (OEG) was reported to form an amphiphilic phospholipid-mimicking prodrug with the anticancer drug camptothecin (13). The nature and the dimensions (eight repeating units) of the oligomer were selected as the watersoluble part to maximize the drug-loading content and also to lower the critical vesicle formation concentration. In this case, a linker characterized by the presence of a thioester bond was used because of its ability of being easily hydrolyzed by esterases, which are abundant in cells. This conjugate was demonstrated to form NPs with a diameter of $\sim 180 \mathrm{~nm}$ and a negative surface charge. The in vitro hydrolysis of the conjugate appeared slow at weakly acidic or neutral $\mathrm{pH}$. Instead, in the presence of esterases, which are abundant in cytoplasm, the conjugate quickly hydrolyzed and released the free drug. Therefore, OEGcamptothecin conjugate was confirmed to act as a prodrug for intracellular release of camptothecin [15].

\section{Hyaluronic acid}

Xin et al. reported the use of hyaluronic acid (HA, 4) as a self-assembly inducer for the formation of three types of paclitaxel-containing NPs. HA is a linear polysaccharide widely distributed throughout connective, epithelial and neural tissues. It is composed of two alternating units of D-glucuronic acid and $\mathrm{N}$-acetyl-D-glucosamine. HA can be easily used as a self-assembly inducer because of its biocompatibility and biodegradability. In addition, it has a strong affinity to cell-specific surface markers overexpressed on the surface of many types of cancer cells, such as glycoprotein CD44 and receptor for HA-mediated motility. Furthermore, in this study different amino acids, such as valine, leucine and phenylalanine, 
were used as spacers between the drug and the carrier to improve the release of paclitaxel from the conjugates. Amino acids are easily used as linkers owing to their bifunctional nature; in fact, they are characterized by the presence of a reactive carboxylic group to conjugate with the drug via an ester bond and the amino group that can be exploited for the linkage with the carrier. It was demonstrated that these conjugates with different aminoacidic linkers were able to self-assemble to form NPs characterized by a diameter of $275-285 \mathrm{~nm}$ [16].

\section{Heparin}

In 2009, valine, leucine and phenylalanine [s3]were used as spacers in another study in which the self-assembly inducer was heparin (5). Heparin is a biocompatible, biodegradable, and water-soluble natural polysaccharide with a structure consisting of variable sulfated repeating disaccharide units. Heparin has different biological activities including anticoagulant activity, inhibition of tumor development and angiogenesis. The synthesis of paclitaxel-heparin conjugates was reported and it was confirmed that they self-assembled in aqueous solution, forming approximately spherical NPs with a diameter of $140-180 \mathrm{~nm}$ composed of a paclitaxel core and a carrier shell [17].

\section{Squalene}

Squalene is a precursor of cholesterol biosynthesis and it belongs to the terpenoid family. Its inertness and biocompatibility justify the use of squalene derivatives $(6)[3,6,18-35]$ and terpenes in general [36-39] for the preparation of self-assembling bioconjugates, as Couvreur reported in several papers. [s4]Squalene can be easily functionalized at its terminal double-bond owing to its compact conformation in very polar solvents. Indeed, the highly coiled conformation leaves [s5]the internal double-bonds sterically shielded whereas the terminal olefinic links are exposed and thus more reactive. So, the terminal double-bond can be converted into [s6]different functional groups such as aldehyde, carboxylic acid, alcohol or 
amine.

Recently, Couvreur and colleagues reported the formation of self-assembled NPs obtained by conjugation of squalene with two antiangiogenic multitarget tyrosine kinase inhibitors: semaxanib (14) and sunitinib (15) [19]. A pH-sensitive linker between the squalene chain and the active compound was introduced because many solid tumors are characterized by an acidic environment and because the intracellular release of the drug is favored by the acidic $\mathrm{pH}$ of endolysosomes. It was demonstrated that the bioconjugates self-assembled in NPs with narrow polydispersity in aqueous solution and an average diameter of $120-140 \mathrm{~nm}$.

The introduction of a squalene tail was further exploited for the derivatization of known anticancer compounds such as paclitaxel, podophyllotoxin (16), camptothecin, epothilone A (17) and cyclopamine (18) with the inclusion of an alternative linker [40-42]. In this study, the squalene moiety and the active compound were connected by a disulfide-containing linker able to guarantee the drug release inside the cell. Indeed, cancer cells express high levels of detoxifying enzymes such as glutathione-S-transferases (GSTs) and glutathione (GSH) to protect themselves from toxic xenobiotics. Testing NPs in the presence of GSH, a progressive slight increase of size was evident and release (moderate) of the free drug was observed.

\section{Amphiphilic drug-drug conjugate}

An innovative self-delivery system in which drugs can be delivered by themselves without any carrier was developed by Huang et al. An amphiphilic drug-drug conjugate, consisting of the hydrophilic anticancer drug irinotecan (19) and hydrophobic anticancer drug chlorambucil (20) has been synthesized and demonstrated to be able to self-assemble in water to form NPs with a diameter of about $88 \mathrm{~nm}$. As a result of the presence of an ester bond between the two drugs, the amphiphilic conjugate was able to release both the compounds after the hydrolysis of this bond in the tumor cells [43]. The same strategy was exploited in a recent study in which the hydrophilic gemcitabine (21) [s7] and the hydrophobic chlorambucil were linked via a hydrolyzable ester bond. The conjugate obtained was used to form NPs following 
the nanoprecipitation technique [10] and it was demonstrated to form NPs [44].

\section{Drugs: modification and biological evaluation}

Different anticancer compounds were used for the formation of self-assembled nanostructures by exploiting the presence of the available functional groups for the linkage with the self-assembly inducer. Paclitaxel (7) is a powerful antitumor agent mainly used for the treatment of breast and ovarian cancer. It inhibits cell replication in the late G2/M phase of the cell cycle by interfering with the depolymerization of microtubules. However, its poor water solubility and toxicity are disadvantages in the use of paclitaxel in cancer treatment. Paclitaxel has been functionalized at the 2' position forming an ester bond, able to be easily cleaved to release the free drug. NPs obtained from the conjugate of paclitaxel with HPPE [8] were biologically evaluated on MCF-7 (breast cancer) and Tca8113 (oral squamous carcinoma) cell lines and they showed potent cellular growth inhibition abilities (Table 1). This result indicated that NPs are able to enter the cell and it is conceivable that the acidic $\mathrm{pH}$ and the enzymes inside the cell would lead to a slow release of paclitaxel which is responsible for the therapeutic effect. In vivo evaluation was made using two malignant tumor cell lines of MCF-7 and Tca8113 to establish tumor models. These evaluations showed that NPs exhibit antitumor effect with a lower toxic effect with respect to the free drug. When paclitaxel was linked to HA using different aminoacidic linkers [16], the obtained NPs were evaluated in vitro (MCF-7 cell line). It was demonstrated that NPs are more cytotoxic than free paclitaxel and flow cytometry analysis showed that NPs significantly enhance the extent of apoptosis-induced cell death. The same authors reported the formation of a conjugate with paclitaxel and heparin [17]. Also in this case, they demonstrated the activity of NPs on MCF-7 cells showing a better activity for the NPs than for the free drug. In vivo evaluation demonstrated that NPs show a similar ovarian tumor growth inhibition to paclitaxel and induce no bodyweight loss. The conjugation of paclitaxel with a squalenic tail through a disulfide-containing linker led to the formation of NPs that were biologically evaluated on MCF-7 cells [40]. It was demonstrated that the conjugation to 
squalene decreased the cytotoxicity of the drug toward MCF-7 cells but it was confirmed that the disulfide bond improved the release of the toxic moiety.

Cisplatin has been widely used in the clinic to treat a variety of cancers such as ovarian, breast and small-cell lung cancer because of its potent activity to crosslink DNA upon entering the cells. However, cisplatin is vulnerable to attack by plasma proteins that deactivate the drug, leading to less therapeutic efficacy, and account for some severe side effects of cisplatin therapy. To improve the therapeutic index of cisplatin while minimizing its adverse side effects, cisplatin analog Pt(IV) prodrugs have been synthesized. Recently the functionalization of a cisplatin analog (9) with a PEG-polylactide copolymer that was covalently bonded to the hydroxylic groups of compound 9 was reported [11]. In vitro evaluation in the A2780 human ovarian carcinoma cell line showed an approximate sevenfold cytotoxicity increase for NPs with cisplatin analog 9 and this result could be due to the burst drug release in the acidic intracellular environment.

Chlorine e6 (10) is an attractive photodynamic therapy drug candidate because of its high absorption in the red spectral region and its cheapness compared with other porphyrin-based photodynamic therapy drugs. Other advantages of chlorine e6 are its long lifetimes in photoexcited triplet states and high molar absorption in the red region of the visible spectrum. Chlorine e6 was recently used for the synthesis of a biocompatible conjugate by the linkage with a polysaccharide exploiting a $\mathrm{pH}$-sensitive linker for the release [12]. Biological evaluation of NPs on HeLa (cervical adenocarcinoma) cells demonstrated that higher levels of apoptosis are induced at $\mathrm{pH} 6.8$ and 6.4 than at $\mathrm{pH} 7.4$, whereas no noticeable difference in cell apoptosis with changes in $\mathrm{pH}$ values was observed in the presence of free chlorine e6. Beclin-1 (11) is the peptide encoded by the human gene BECN1 and participates in autophagy regulation. It has been suggested that the overexpression of beclin-1 could inhibit tumor development but this peptide has some drawbacks. In fact, beclin-1 has a low chemical stability in vivo and it is characterized by a nonspecific biodistribution in tissues. To overcome these disadvantages, beclin-1 was engineered to have a thiol group at the $\mathrm{N}$ terminus to be easily conjugated with a $\mathrm{pH}$-sensitive polymer [13]. The obtained NPs were biologically evaluated on MCF-7 cells and they showed significant cytotoxicity. It was also proved that NPs strongly induced autophagy and potentially led to autophagic cell death. In 
vivo evaluation on MCF-7 cancer cells in a [s8]xenografted tumor nude mouse model demonstrated that the tumor size of tumor-bearing nude mice treated with NPs increased more slowly than that of the control group, demonstrating that NPs can kill tumor cells synergistically and effectively showing no extreme toxicity.

Doxorubicin (12), a widely used anticancer drug, was involved in the formation of a dual pHsensitive polymer conjugate [14]. Doxorubicin was linked to PEG through an acid-labile hydrazone bond. SK-3rd, a cancer stem cell line, was chosen to evaluate the efficacy of doxorubicin NPs and it was demonstrated that NPs enhanced the cellular internalization, subsequently released the free drug in the cells in response to the endo/lysosomal $\mathrm{pH}$ and thus inhibited the progression of cancer stem cells. Moreover Song et al. described the synthesis of a disulfide-linked doxorubicin drug-drug conjugate [45]. The in vitro cytotoxicity of doxorubicin NPs was weaker than that of the free drug but the antitumor efficacy of NPs evaluated in MCF-7-bearing mice was demonstrated to be higher than that of free doxorubicin.

In 2010, the hydrophobic anticancer drug camptothecin[s9] (13) was involved in the formation of an amphiphilic conjugate with a short ethylene glycol [15]. In addition, these NPs were loaded with the water-soluble drug doxorubicin to obtain a synergic effect. The cytotoxicity of these NPs was evaluated in SKOV-3 ovarian and MCF-7 breast cancer cell lines. Camptothecin-NPs were shown to be as cytotoxic as the free drug in the cell lines but doxorubicin-loaded NPs showed a higher activity than camptothecin-NPs, free camptothecin and doxorubicin demonstrating an additive or synergic anticancer activity of the two drugs involved in the NPs. In vivo evaluations were performed using athymic mice bearing intraperitoneal tumors and camptothecin-NPs were demonstrated to have a strong in vivo anticancer activity.

Betulinic acid (8) is an anticancer compound that has some drawbacks such as poor water solubility, severe side effects for healthy tissues, rapid blood clearance and low tumor selectivity. However, the formulation of betulinic acid with [s10]NPs can overcome these problems. In particular, the conjugation with PEG induced the formation of NPs that were also loaded with the anticancer drug hydroxycamptothecin (22) [9]. The cytotoxicity of these NPs was evaluated on Lewis lung carcinoma (LLC) and A549 (human lung cancer) cell lines 
and it was considerably higher than that seen with free betulinic acid. The combination therapy of NPs containing betulinic acid and hydroxycamptothecin results in a better in vitro activity than the two free drugs, suggesting a significant synergic effect of NPs by codelivering of the two drugs. In vivo efficacy of NPs was evaluated in xenograft models of lung tumors - tumor-bearing mice treated with NPs showed a clear survival advantage compared with the control-treated mice. The antitumor effect of NPs was higher than the ones for free betulinic acid and hydroxycamptothecin, and no signs of systemic toxicity were observed by monitoring bodyweight of mice. Moreover, the authors reported the preparation of NPs with the addition of a target molecule (folate). These NPs were demonstrated to be even more active in vitro and in vivo with respect to the NPs with no target molecules, confirming that these NPs could be delivered into cells more easily because of the presence of the folate.

Semaxanib (14) and sunitinib (15) are multitarget tyrosine kinase inhibitors but they have some side effects such as cardiotoxicity, severe cutaneous toxicities and hematotoxicity. The NPs obtained by the covalent linkage of these compounds with squalene were biologically evaluated on human umbilical vein endothelial cells (HUVECs), demonstrating that squalenoyl-sunitinib conjugate NPs showed notable cytotoxicity whereas semaxanib NPs were not active [19]. In addition, sunitinib NPs showed moderate cytotoxic activity against Mia Paca2 pancreatic cancer cells.

The water-soluble drug irinotecan (19), a camptothecin derivative and a potent DNA topoisomerase I inhibitor, and the water-insoluble chlorambucil (20), a DNA alkylating anticancer drug, were used to form an amphiphilic conjugate by an esterification reaction [43]. The NPs obtained were biologically evaluated using MCF-7 and HeLa cell lines. The cytotoxic activity of the drug-drug conjugate was demonstrated to be strongly dependent on its concentration - if the concentration is lower than the CAC value the cytotoxic activity is worse than the mixture of the two drugs but if the concentration is higher than the CAC value NPs showed a much better activity than the free drug mixture. This result suggested that the NPs enter the tumor cells and that the released irinotecan and chlorambucil have a synergic action. Furthermore, MCF-7 tumor-bearing mice were intravenously injected with irinotecan, chlorambucil, irinotecan/chlorambucil mixture and irinotecan/chlorambucil NPs; at the end of the experiments the tumor volumes in mice treated with NPs were much smaller than the 
tumor volumes of all other mice, demonstrating that NPs produce a better tumor growth inhibition than the free drugs and their mixture. Moreover the hydrophilic drug irinotecan was recently used with the hydrophobic combretastatin A-4 to form a drug-drug conjugate. The obtained NPs were evaluated in MCF-7 and HUVEC cell lines and they showed a reduced cell survival ratio [46].

The use of chlorambucil in combination with another hydrophilic anticancer compound (gemcitabine, 21) was also reported in 2015 [44]. In vitro evaluation of NPs toward a number of cancer cell lines [SMMC-7721 (human hepatoma), HeLa, MCF-7, SGC-7901 (gastric cancer), U87 (glioblastoma)] confirmed an increased cytotoxic activity with respect to the free drugs. Imaging studies were performed and it was demonstrated that NPs are internalized in the cell. The in vivo anticancer activity of NPs was evaluated in the SMMC-7721 tumorbearing mouse model and it was reported that NPs have a high anticancer efficacy probably owing to the tumor targeting via the EPR effect. No significant bodyweight loss was observed, showing that NPs are well tolerated at the tested dose level.

It is worth highlighting the possibility to obtain hetero-NPs by mixing different drugs functionalized with the same self-assembly inducer. Couvreur and colleagues reported the formation of hetero-NPs by co-self-assembly of three squalene-based conjugates: gemcitabine-squalene (therapeutic component), rhodamine-squalene (fluorescent moiety) and biotin-squalene (targeting agent) [26]. These hetero-NPs demonstrated improved internalization in MCF7, M109 (murine lung cancer) and HeLa cell lines, which overexpress biotin receptors, and a stronger cytotoxic activity than nonbiotinylated NPs.

Our research group studied the formation of hetero-NPs by co-self-assembly of two squalene-based conjugates with the drugs paclitaxel (7) and cyclopamine (18) [42]. Biological evaluation of hetero-NPs on OVCAR5 (ovarian cancer), \#83 and \#110 (ovarian tumor-initiating), and U251 (glioblastoma) cell lines demonstrated a combined efficacy in apoptosis induction. Furthermore, we reported the formation of fluorescent hetero-NPs by coself-assembly of the above-mentioned drug-squalene conjugates with a tetramethylrhodamine-squalene conjugate. The demonstration of cell internalization was accomplished by the application of multiple fluorescence microscopy methods - three- 
dimensional confocal microscopy allowed the identification of fluorescent NPs in the cytoplasm of the cells and super-resolution microscopy (dSTORM) permitted calculation of the diameters of the bright particles.

\section{Concluding remarks}

This review has highlighted the recent progress in the field of prodrug conjugates able to form NPs by self-assembly. The simple functionalization of known anticancer compounds and the easy formation of NPs make this strategy simple to apply for different types of drugs and biological targets. This approach could improve drug biocompatibility and delivery efficacy. In addition, the functionalization of different known drugs with a cleavable linker and a proper lipophilic chain that induces self-assembly and the generation of hetero-NPs could be exploited for personalized treatment of different types of diseases [42-44]. The same strategy can be used for the preparation of fluorescent hetero-NPs that can help follow the dynamics of the delivery and it might be possible to trace these NPs in the cells by imaging $[26,40]$.

\section{Acknowledgments}

This work has been developed under the umbrella of CM1106 COST Action 'Chemical Approaches for Targeting Drug Resistance in Cancer Stem Cells' (http://www.stemchem.org) and was supported by MIUR - University of Turin 'Fondi Ricerca Locale (ex-60\%)'.

\section{References}

1 Brigger, I. et al. (2012) Nanoparticles in cancer therapy and diagnosis. Adv. Drug Deliv. Rev. 64, 24-36

2 Talekar, M. et al. (2011) Targeting of nanoparticles in cancer: drug delivery and diagnostics. Anticancer Drugs 22, 949-962

3 Hillaireau, H. and Couvreur, P. (2009) Nanocarriers' entry into the cell: relevance to drug delivery. Cell. Mol. Life Sci. 66, 2873-2896 
4 Mura, S. et al. (2015) Lipid prodrug nanocarriers in cancer therapy. J. Control. Release 208, 25-41

5 Delplace, V. et al. (2014) Recent trends in the design of anticancer polymer prodrug nanocarriers. Polymer Chemistry 5, 1529-1544

6 Bildstein, L. et al. (2011) Prodrug-based intracellular delivery of anticancer agents. Adv. Drug Deliv. Rev. 63, 3-23

7 Cho, E.J. et al. (2013) Nanoparticle characterization: state of the art, challenges, and emerging technologies. Molecular Pharmaceutics 10, 2093-2110

8 Li, G. et al. (2011) Polymeric micelles with water-insoluble drug as hydrophobic moiety for drug delivery. Biomacromolecules 12, 2016-2026

9 Dai, L. et al. (2015) Self-assembled targeted folate-conjugated eight-arm-polyethylene glycol-betulinic acid nanoparticles for co-delivery of anticancer drugs. J. Mater. Chem. B 3, 3754-3766

10 Lepeltier, E. et al. (2014) Nanoprecipitation and the "Ouzo effect": application to drug delivery devices. Adv. Drug Deliv. Rev. 71, 86-97

11 Aryal, S. et al. (2010) Polymer-cisplatin conjugate nanoparticles for acid-responsive drug delivery. Acs Nano 4, 251-258

12 Park, S.Y. et al. (2011) A smart polysaccharide/drug conjugate for photodynamic therapy. Angew. Chem. Int. Ed. Engl. 50, 1644-1647

13 Yi, W. et al. (2015) Self-assembled autophagy-inducing polymeric nanoparticles for breast cancer interference in-vivo. Adv. Mater. 27, 2627-2634

14 Du, J-Z. et al. (2011) Tailor-made dual pH-sensitive polymer-doxorubicin nanoparticles for efficient anticancer drug delivery. J. Am. Chem. Soc. 133, 1756017563

15 Shen, Y. et al. (2010) Prodrugs forming high drug loading multifunctional nanocapsules for intracellular cancer drug delivery. J. Am. Chem. Soc. 132, 42594265 
16 Xin, D. et al. (2010) The use of amino acid linkers in the conjugation of paclitaxel with hyaluronic acid as drug delivery system: synthesis, self-assembled property, drug release, and in vitro efficiency. Pharm. Res. 27, 380-389

17 Wang, Y. et al. (2009) Heparin-paclitaxel conjugates as drug delivery system: synthesis, self-assembly property, drug release, and antitumor activity. Bioconj. Chem. 20, 2214-2221

18 Bekkara-Aounallah, F. et al. (2008) Novel PEGylated nanoassemblies made of selfassembled squalenoyl nucleoside analogues. Advanced Functional Materials 18, $3715-3725$

19 Buchy, E. et al. (2015) Synthesis and cytotoxic activity of self-assembling squalene conjugates of 3-(pyrrol-2-yl)methylidene-2,3-dihydro-1H-indol-2-one anticancer agents. European Journal Organic Chemistry 1, 202-212

20 Caron, J. et al. (2010) Squalenoyl nucleoside monophosphate nanoassemblies: new prodrug strategy for the delivery of nucleotide analogues. Bioorg. Med. Chem. Lett. $20,2761-2764$

21 Caron, J. et al. (2011) Squalenoyl gemcitabine monophosphate: synthesis, characterisation of nanoassemblies and biological evaluation. European Journal Organic Chemistry 14, 2615-2628

22 Caron, J. et al. (2013) Improving the antitumor activity of squalenoyl-paclitaxel conjugate nanoassemblies by manipulating the linker between paclitaxel and squalene. Advanced Healthcare Materials 2, 172-185

23 Couvreur, P. et al. (2006) Squalenoyl nanomedicines as potential therapeutics. Nano Lett. 6, 2544-2548

24 Desmaele, D. et al. (2012) Squalenoylation: a generic platform for nanoparticular drug delivery. J. Control. Release 161, 609-618

25 Dosio, F. et al. (2010) Novel nanoassemblies composed of squalenoyl-paclitaxel derivatives: synthesis, characterization, and biological evaluation. Bioconj. Chem. 21, 
$1349-1361$

26 Duc Trung, B. et al. (2014) Multifunctional squalene-based prodrug nanoparticles for targeted cancer therapy. Chemical Communications 50, 5336-5338

27 Hillaireau, H. et al. (2013) Anti-HIV efficacy and biodistribution of nucleoside reverse transcriptase inhibitors delivered as squalenoylated prodrug nanoassemblies. Biomaterials 34, 4831-4838

28 Maksimenko, A. et al. (2013) Polyisoprenoyl gemcitabine conjugates self assemble as nanoparticles, useful for cancer therapy. Cancer Lett. 334, 346-353

29 Raouane, M. et al. (2011) Synthesis, characterization, and in vivo delivery of siRNAsqualene nanoparticles targeting fusion oncogene in papillary thyroid carcinoma. $J$. Med. Chem. 54, 4067-4076

30 Reddy, L.H. et al. (2008) Oral absorption and tissue distribution of a new squalenoyl anticancer nanomedicine. Journal Nanoparticle Research 10, 887-891

31 Reddy, L.H. et al. (2008) Squalenoylation favorably modifies the in vivo pharmacokinetics and biodistribution of gemcitabine in mice. Drug Metab. Dispos. 36, $1570-1577$

32 Reddy, L.H. et al. (2008) Preclinical toxicology (subacute and acute) and efficacy of a new squalenoyl gemcitabine anticancer nanomedicine. J. Pharmacol. Exp. Ther. 325, 484-490

33 Reddy, L.H. and Couvreur, P. (2009) Squalene: a natural triterpene for use in disease management and therapy. Adv. Drug Deliv. Rev. 61, 1412-1426

34 Semiramoth, N. et al. (2012) Self-assembled squalenoylated penicillin bioconjugates: an original approach for the treatment of intracellular infections. Acs Nano 6, 38203831

35 Valetti, S. et al. (2014) Peptide-functionalized nanoparticles for selective targeting of pancreatic tumor. J. Control. Release 192, 29-39

36 Caron, J. et al. (2014) Combined antitumoral therapy with nanoassemblies of 
bolaform polyisoprenoyl paclitaxel/gemcitabine prodrugs. Polymer Chemistry 5, 1662-1673

37 Duc Trung, B. et al. (2013) Polymer prodrug nanoparticles based on naturally occurring isoprenoid for anticancer therapy. Biomacromolecules 14, 2837-2847

38 Mura, S. et al. (2013) Novel isoprenoyl nanoassembled prodrug for paclitaxel delivery. Bioconj. Chem. 24, 1840-1849

39 Maksimenko, A. et al. (2014) A unique squalenoylated and nonpegylated doxorubicin nanomedicine with systemic long-circulating properties and anticancer activity. Proc. Natl. Acad. Sci. U. S. A. 111, E217-226

40 Borrelli, S. et al. (2014) New class of squalene-based releasable nanoassemblies of paclitaxel, podophyllotoxin, camptothecin and epothilone A. European Journal Medicinal Chemistry 85, 179-190

41 Borrelli, S. et al. (2015) Self-assembled squalene-based fluorescent heteronanoparticles. ChemPlusChem 80, 47-49

42 Fumagalli, G. et al. (2015) Cyclopamine-paclitaxel-containing nanoparticles: internalization in cells detected by confocal and super-resolution microscopy. ChemPlusChem 80, 1380-1383

43 Huang, P. et al. (2014) Combination of small molecule prodrug and nanodrug delivery: amphiphilic drug-drug conjugate for cancer therapy. J. Am. Chem. Soc. 136, $11748-11756$

44 Fan, M. et al. (2015) Chlorambucil gemcitabine conjugate nanomedicine for cancer therapy. European Journal Pharmaceutical Sciences 79, 20-26

45 Song, Q. et al. (2016) Reduction responsive self-assembled nanoparticles based on disulfide-linked drug-drug conjugate with high drug loading and antitumor efficacy. Mol. Pharm. 13, 190-201

46 Zhang, R. et al. (2016) Hypoxia-responsive drug-drug conjugated nanoparticles for breast cancer synergistic therapy. RSC Advances 6, 30268-30276 


\section{Figure legends}

Figure 1. Structures of self-assembling inducers used for the formation of the conjugates. The anchor point used for the conjugation reaction with the active compound is highlighted in red.

Figure 2. Structures of anticancer compounds used as building blocks for the formation of the conjugate compounds. The anchor point used for the conjugation reaction with the self-assembling inducer is highlighted in red. 
Table 1. Nanoparticle properties

\begin{tabular}{|c|c|c|c|c|c|c|c|}
\hline Drug & Linker & $\begin{array}{l}\text { Self-assembly } \\
\text { inducer }\end{array}$ & $\begin{array}{l}\text { Diameter } \\
(\mathrm{nm})\end{array}$ & $\begin{array}{l}\text { Z potential } \\
(\mathrm{mV})\end{array}$ & Cell line & $\begin{array}{l}\text { In vivo } \\
\text { evaluation }\end{array}$ & $\begin{array}{c}\text { Ref } \\
s\end{array}$ \\
\hline 9 & Hydrazone bond & 2 & $\sim 86$ & -33 & A2780 & & [11] \\
\hline Beclin-1 (11) & $\begin{array}{l}\text { 3-(Dibutylamino)- } \\
\text { 1-propylamine }\end{array}$ & 2 & $\sim 32$ & +24 & MCF-7 & $\checkmark$ & [13] \\
\hline $\begin{array}{l}\text { Betulinic acid (8) } \\
\text { Hydroxycamptothecin (22) }\end{array}$ & 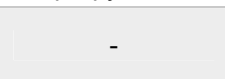 & 2 & $108-125$ & $\begin{array}{c}+5.3 \text { to } \\
{[\mathrm{s} 11]+86}\end{array}$ & LLC or A549 & $\checkmark$ & [9] \\
\hline Camptothecin[s12] (13) & $\beta$-Thioester & 2 & $\sim 186$ & -4 & $\begin{array}{l}\text { SKOV-3 } \\
\text { MCF-7 }\end{array}$ & $\checkmark$ & [15] \\
\hline Camptothecin (13) & Disulfide & 6 & $\sim 195$ & -41 & MCF-7 & & [40] \\
\hline $\begin{array}{l}\text { Chlorambucil (20) } \\
\text { Gemcitabine (21) }\end{array}$ & - & - & $\sim 160$ & +15.7 & $\begin{array}{c}\text { SMMC-7721 } \\
\text { Hela } \\
\text { MCF-7 } \\
\text { SGC-7901 } \\
\text { U87 }\end{array}$ & $\checkmark$ & [44] \\
\hline $\begin{array}{l}\text { Chlorambucil (20) } \\
\text { Irinotecan (19) }\end{array}$ & - & - & $\sim 88$ & +3.4 & $\begin{array}{l}\text { MCF-7 } \\
\text { HeLa }\end{array}$ & $\checkmark$ & [43] \\
\hline Chlorine e6 (10) & $\begin{array}{c}\text { 3-Diethyl } \\
\text { aminopropyl } \\
\text { isothiocyanate }\end{array}$ & $2+3$ & $\sim 150$ & -8 & HeLa & $\checkmark$ & [12] \\
\hline Cyclopamine (18) & Disulfide & 6 & $\sim 105$ & +37 & U251 & & [42] \\
\hline $\begin{array}{l}\text { Cyclopamine (18) } \\
\text { Paclitaxel (7) }\end{array}$ & Disulfide & 6 & $\sim 229$ & +26 & U251 & & [42] \\
\hline Doxorubicin (12) & - & 6 & $\sim 130$ & +35 & $\begin{array}{l}\text { Mia PaCa-2 } \\
\text { M109 }\end{array}$ & $\checkmark$ & [39] \\
\hline Doxorubicin (12) & Hydrazone bond & 2 & $\sim 27$ & n.a. & SK-3rd & & [14] \\
\hline Doxorubicin (12) & Disulfide & - & $\sim 89$ & n.a. & MCF-7 & $\checkmark$ & [45] \\
\hline Epothilone A (17) & Disulfide & 6 & $\sim 161$ & -56 & MCF-7 & & [40] \\
\hline Gemcitabine (21) & - & 6 & $\sim 130$ & n.a. & $\begin{array}{c}\text { MCF-7 } \\
\text { KB3-1 } \\
\text { L1210 10K }\end{array}$ & $\checkmark$ & [23] \\
\hline Gemcitabine (21) & - & 6 & $\sim 149$ & -25 & $\begin{array}{l}\text { HeLa } \\
\text { M109 } \\
\text { MCF7 }\end{array}$ & & [26] \\
\hline Gemcitabine (21) & - & 6 & $130-170$ & -6 & Pancreatic & $\checkmark$ & [35] \\
\hline Gemcitabine (21) & - & 6 & $\sim 104$ & -6 & L1210 10K & & [18] \\
\hline $\begin{array}{c}\text { Gemcitabine } \\
\text { monophosphate (23) }\end{array}$ & - & 6 & $50-150$ & n.a. & L1210 $10 \mathrm{~K}$ & & [21] \\
\hline Irinotecan (19) & $\begin{array}{l}\text { Azobenzene } \\
\text { bond }\end{array}$ & - & $\sim 196$ & n.a. & $\begin{array}{l}\text { MCF-7 } \\
\text { HUVEC }\end{array}$ & $\checkmark$ & [46] \\
\hline Paclitaxel (7) & Val, Leu, Phe & 4 & $275-285$ & $\begin{array}{c}+0.17 \text { to } \\
{[\mathrm{s} 13]+0.43}\end{array}$ & MCF-7 & & [16] \\
\hline Paclitaxel (7) & Val, Leu, Phe & 5 & $140-180$ & $\begin{array}{l}-21 \text { to } \\
-31[\mathrm{~s} 14]\end{array}$ & MCF-7 & $\checkmark$ & [17] \\
\hline Paclitaxel (7) & - & 1 & $50-120$ & n.a. & $\begin{array}{l}\text { MCF-7 } \\
\text { Tca8113 }\end{array}$ & $\checkmark$ & [8] \\
\hline Paclitaxel (7) & Disulfide & 6 & $\sim 103$ & -37 & MCF-7 & & [40] \\
\hline Paclitaxel (7) & cis, cis-1,4-dienic & 6 & $90-150$ & $\begin{array}{c}-19 \text { to } \\
-[\mathrm{s} 15] 26\end{array}$ & $\begin{array}{c}\text { A549 } \\
\text { HT-29 } \\
\text { KB } 3.1\end{array}$ & $\checkmark$ & [22] \\
\hline Paclitaxel (7) & Ester bond & 6 & $118-293$ & $\begin{array}{c}-44 \text { to } \\
-36[\mathrm{~s} 16]\end{array}$ & M109 & & [25] \\
\hline Paclitaxel (7) & - & 6 & $149-225$ & n.a. & A549 & & [41] \\
\hline Podofillotoxin (16) & Disulfide & 6 & $\sim 184$ & -11 & MCF-7 & & [40] \\
\hline Semaxanib (14) & Hemiaminal & 6 & $\sim 141$ & -40 & HUVEC & & [19] \\
\hline siRNA (24) & $\begin{array}{l}\text { Maleimide- } \\
\text { sulfhydryl }\end{array}$ & 6 & $\sim 165$ & -26 & $\begin{array}{c}\text { TPC-1 } \\
\text { BHP 10-3 }\end{array}$ & $\checkmark$ & [29] \\
\hline Sunitinib (15) & Hemiaminal & 6 & $\sim 123$ & +23 & $\begin{array}{l}\text { HUVEC } \\
\text { Mia Paca2 }\end{array}$ & & [19] \\
\hline
\end{tabular}

Abbreviation: n.a., not available[s17]. 


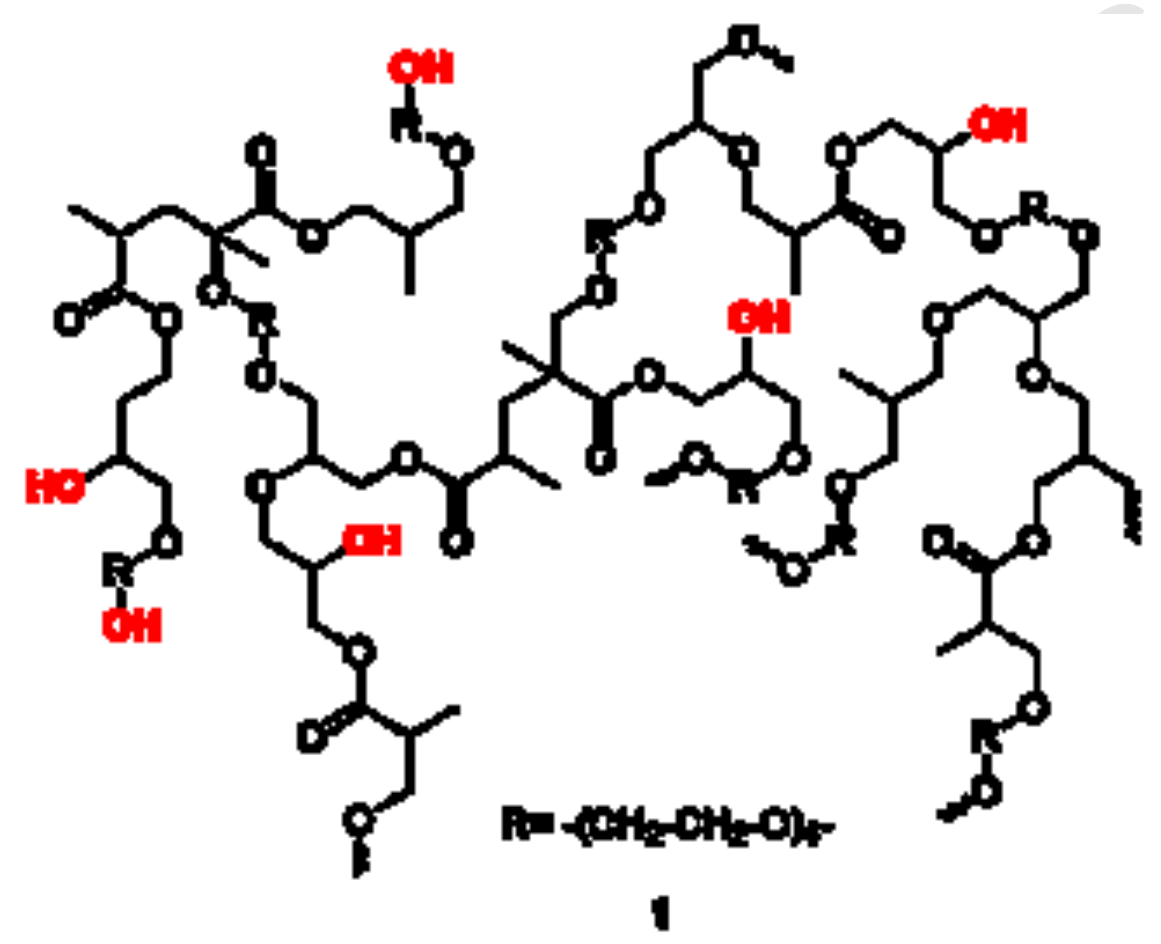

(to.
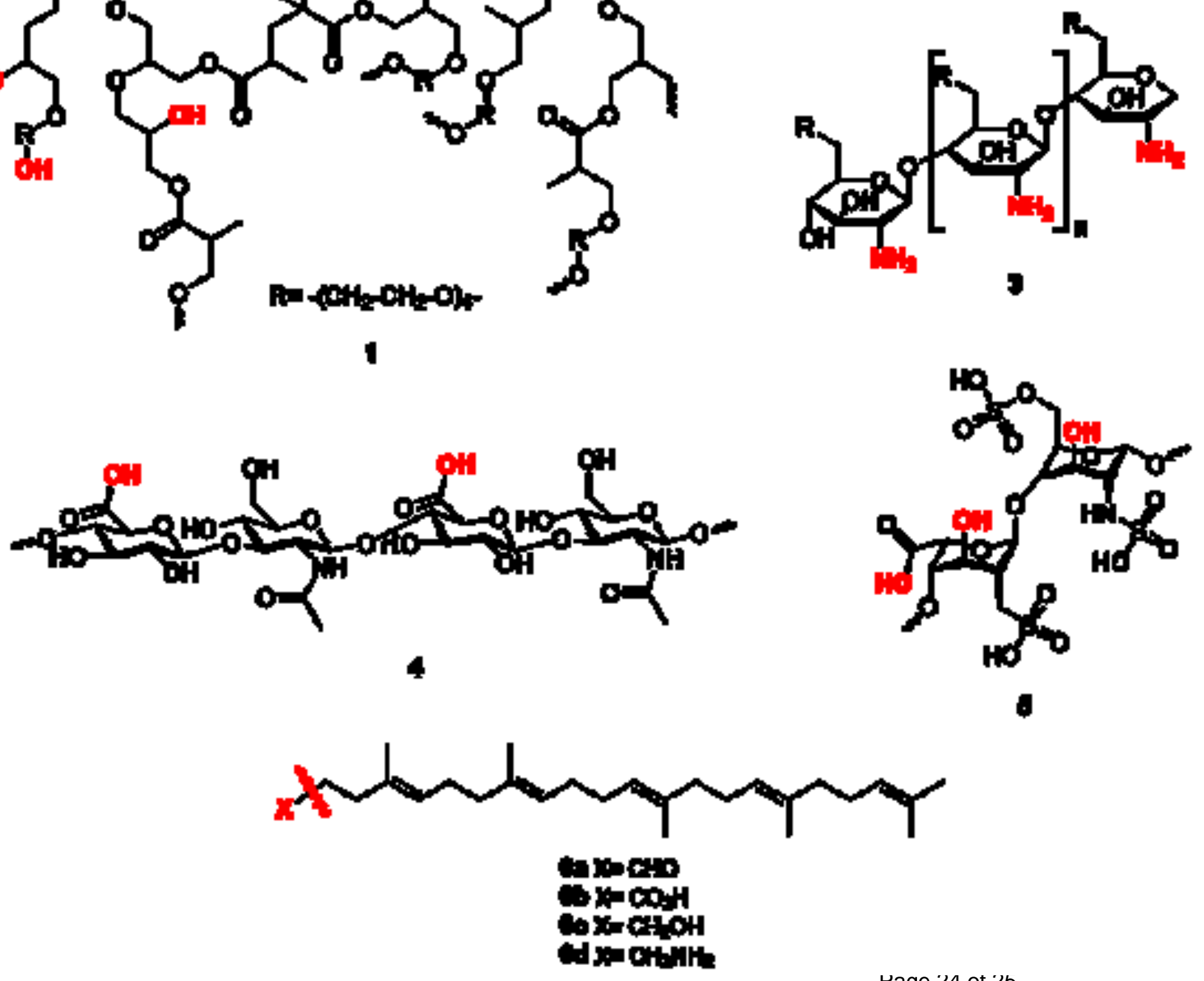


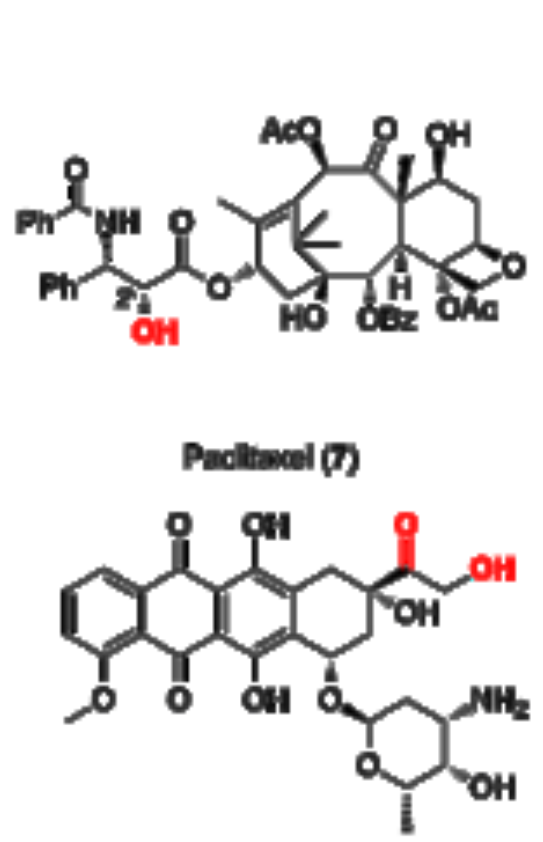

Dosorubidin (12)

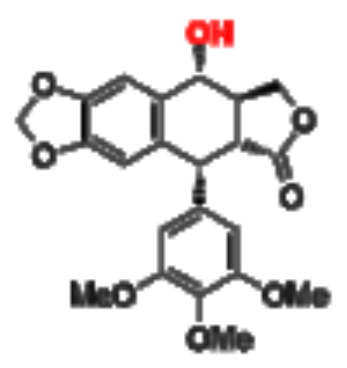

Podophylloloxin (16) (n)

Bonlinie acid (a)

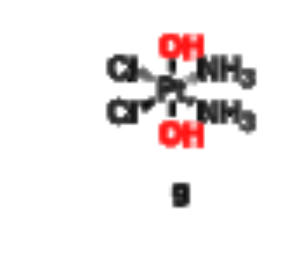

Beclin-1 (T) (proteli -450 an ac)

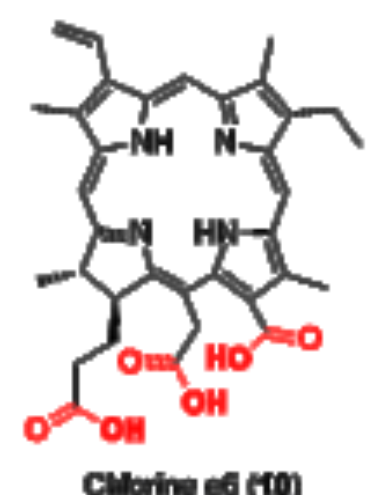

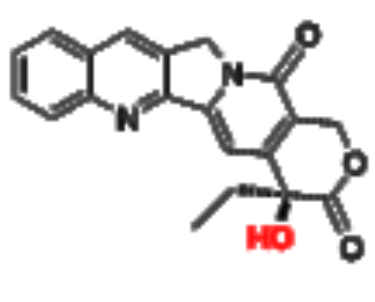

Caaphoflecta (13)

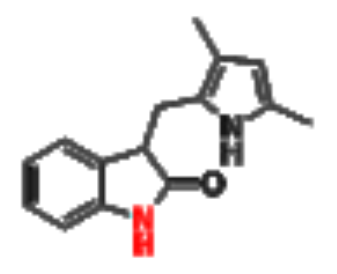

Senaxanilb (14)

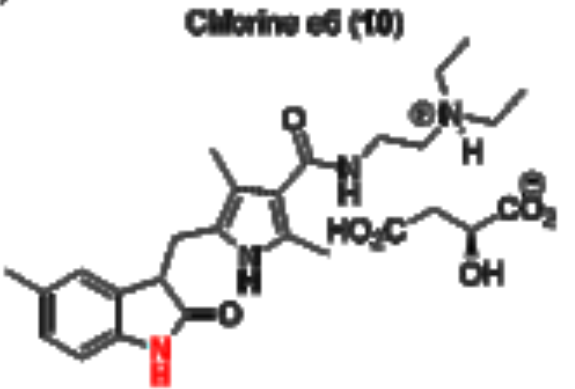

Sesultialb Lenulate sedil (15)

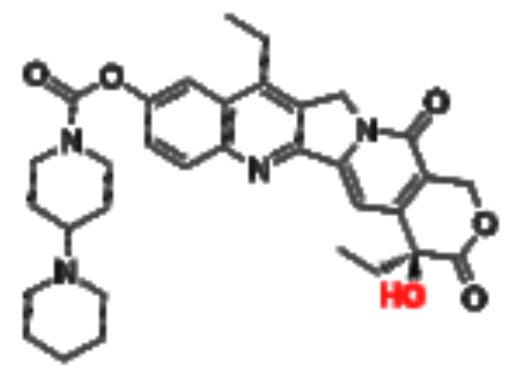

Inotecan (19)

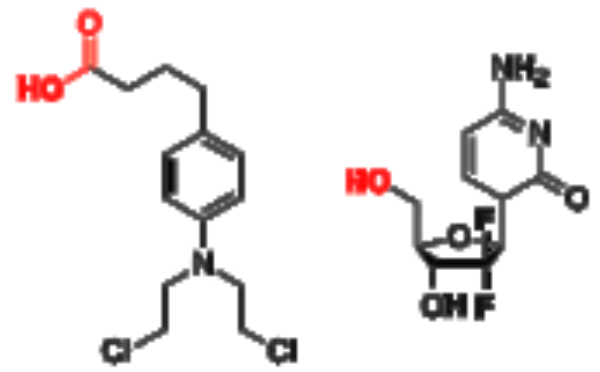

Calorantudel (20)

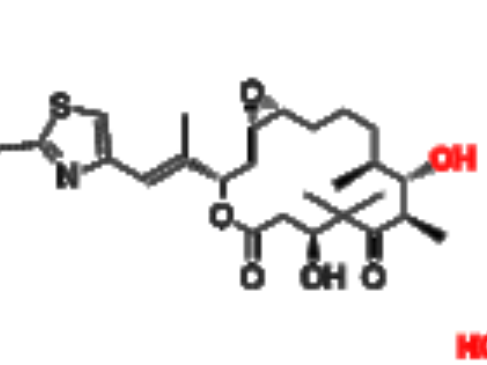

Epothllone A (17)

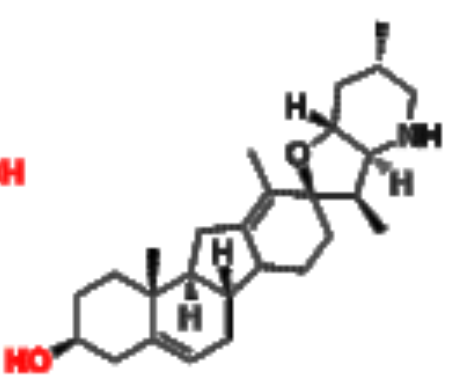

Cxcbpiniline (t9)

$$
\text { C:OH }
$$

